\title{
BIO-CONTROL ACTIVITY OF BACTERIAL STRAINS ON POSTHARVEST PERFORMANCE OF Gladiolus L. HYBRIDS 'MAMMOTH'
}

\author{
Iftikhar Ahmad ${ }^{1,2, *}$, Muhammad Saleem ${ }^{2,3}$, John M. Dole ${ }^{2}$ and Ann G. Matthysse ${ }^{4}$ \\ ${ }^{1}$ Institute of Horticultural Sciences, University of Agriculture, Faisalabad-38040, Pakistan; ${ }^{2}$ Department of \\ Horticultural Science, North Carolina State University, Raleigh, NC, 27695, USA; ${ }^{3}$ Department of Horticulture, \\ Bahauddin Zakariya University, Bahadur Sub-campus, Layyah, Pakistan; ${ }^{4}$ Department of Biology, University of \\ North Carolina, Chapel Hill, NC, 27599, USA. \\ "Corresponding author's email: iftikharahmadhashmi@gmail.com, iahmad@uaf.edu.pk
}

\begin{abstract}
A study was carried out to elucidate the efficacy of different bacterial strains on controlling detrimental bacteria and on vase life extension of Gladiolus L. hybrids 'Mammoth'. In a preliminary study, three bacterial strains (Bacillus pumilus, Delftia acidovorans, and Herbasperillum sp.) were isolated from the vase solutions of cut Gladiolus, identified and cultured to obtain the bacteria to be used in the study. These isolated strains were compared with two strains of Pseudomonas fluorescens (PF279 and PF-417). All tested strains produced similar vase life of cut gladiolus stems, which was also similar to that of stems placed in tap water. However, stems placed in solutions with PF-279 Pseudomonas fluorecsens resulted in highest water uptake, while the stems placed in solutions with higher concentrations of Herbasperillum sp. $\left(10 \mathrm{or} 20 \mathrm{~mL} \mathrm{~L}^{-1}\right)$ had lowest water uptake. Cut stems require low $\mathrm{pH}$ for sustaining water uptake and vase life extension, but use of nutrient broth to culture bacteria increased initial solution $\mathrm{pH}$, which resulted in early senescence of cut stems due to rapid bacterial growth, vascular occlusion due to embolisms and reduced stem hydraulic conductance compared to the stems placed in tap water (control). In summary, tested bacterial strains had no effect on controlling detrimental bacteria present in the vase solutions or vase life extension of cut gladiolus and cannot be used in organic floral preservatives for holding cut stems after harvest.
\end{abstract}

Keywords: Pseudomonas fluorescens, Bacillus pumilus, Herbasperillum sp., Delftia acidovorans, beneficial microbes, vase life.

\section{INTRODUCTION}

Gladiolus (Gladiolus L. hybrids), one of the most popular bulbous cut flowers of the world, has the problem of shorter vase life due to vascular occlusion by bacteria. Stem blockage in cut flowers is generally caused by bacterial proliferation along with their decay products (Teixeira-da-Silva, 2003). Bio-control through bacteria represent a potential alternative management approach (Jetiyanon and Kloepper, 2002) and may help in developing an organic method for effectively controlling detrimental microbes in the vase solutions (Carlson et al., 2015). The bio-control agents are used for biological management of pests to control a specific microbe (Shanmugam et al., 2011; Sajjad et al., 2014). Among bacterial antagonists, Pseudomonas fluorescens is most effective against a wide range of plant pathogens infecting different plants such as carnation, bean, radish, cucumber, tomato, and tobacco (van Loon et al., 1998), while P. fulva has extended vase life of cut zinnia stems (Carlson et al., 2015). Moreover, Burkholderia cepacia and Bacillus spp. (spore forming Gram-positive bacteria) have effectively been used to control plant diseases (Kloepper et al., 2004). Use of compatible and multiple bio-control agents in various groups also helps to control plant diseases, such as combinations of bacteria (Raupach and Kloepper, 1998; Shanmugam et al., 2002), fungi (Paulitz et al., 1990), bacteria and fungi (Duffy et al., 1996), yeasts (Janisiewicz, 1996), and bacteria and yeast (Janisiewicz and Bors, 1995).

An experiment was conducted to compare different beneficial bacterial strains for their efficacy to control detrimental bacteria in vase solutions with gladiolus stems. There is dire need to develop organic preservatives for keeping flower organic grown without chemicals until end of vase life. However, there are currently no effective organic preservatives available in the market and those available are not effective (Ahmad et al., 2014). Organic carbohydrate source and acidifier are available but organic biocides are not available. Therefore, this study was conducted to elucidate the effect of bio-control bacterial strains, some of which have been proved effective for various agronomic crops, in controlling detrimental bacteria in vase solutions and effect on the postharvest water relations and quality characteristics of cut gladiolus. Moreover, the findings of the study would help develop an organic floral preservative to be used for handling organically grown cut flowers. 


\section{MATERIALS AND METHODS}

The study was conducted at the Postharvest Laboratory, Department of Horticultural Science, NC State University, Raleigh, NC, during 2012-13. Prior to the study, a preliminary trial was conducted by placing fifteen stems in three vases [containing $500 \mathrm{~mL}$ deionized (DI) water] with five stems per vase. Stems were observed daily for differences in appearance due to senescence. Aliquots of the vase solution were taken on day 7 of vase life (which was when the stems started showing differences in appearance) from each vase, diluted, and cultured on nutrient agar plates to isolate the bacterial strains. On the basis of size and color, three types of colonies were isolated and re-cultured until pure cultures were obtained and submitted to the NCSU Plant Disease and Insect Clinic for identification of strains. Two other strains, Pseudomonas fluorescens (PF-279) and Pseudomonas fluorescens (PF-417), were obtained from Washington State University, WA, USA, and University of North Carolina, Chappel Hill, NC, USA, respectively, and compared along with strains isolated from the vase solutions.

Plant material: Cut stems of gladiolus (Gladiolus L. hybrids 'Mammoth') were received from a commercial grower, Glad-
A-Way Farms, California, USA, within four days of harvest. On arrival, stems were sorted into 19 similar groups on the basis of stem caliper. Initially stems were sorted into groups with similar diameters followed by distribution uniformly among the treatments. Thus, each treatment would contain the same number of thick, intermediate and thin stems. Stems were dipped in soapy solution for $5 \mathrm{sec}$., rinsed in tap water, surface sterilized by spraying $70 \%$ alcohol, recut with sterilized secateurs to final uniform stem length of $80 \mathrm{~cm}$, labeled, and placed in vases (three stems per vase) in a vaselife evaluation room set at $21 \pm 1^{\circ} \mathrm{C}$ temperature, $40-60 \%$ relative humidity $(\mathrm{RH})$ and a $12 \mathrm{~h}$ light period provided by cool-white fluorescent tubes, which provided a photosynthetic photon flux of $\sim 20 \mu \mathrm{mol} \mathrm{m}^{-2} \mathrm{~s}^{-1}$ measured with a 1078 QMSW Quantum meter (Apogee Instruments, Inc., Logan, UT, USA).

Treatments: Inoculum of each strain was prepared by inoculating single colony from isolated pure cultures and grown for $48 \mathrm{hrs}$. with continuous shaking. Vases containing $700 \mathrm{~mL}$ of tap water were sterilized and covered with parafilm to reduce the chances of air contamination. Initial bacterial population varied from $1 \times 10^{4}$ to $25 \times 10^{4} \mathrm{cfu} \mathrm{mL}^{-1}$ for different strains. There were 19 treatments with all five strains

Table 1. Effect of different concentrations of various bacterial strains on vase life and weight changes of 'Mammoth' gladiolus. Stems were placed in either tap water, pure nutrient broth, or in nutrient broth plus Pseudomonas fluorescens (PF-279), Pseudomonas fluorescens (PF-417), Bacillus pumilus, Herbasperillum sp., or Delftia acidovorans, at 5,10 , or $20 \mathrm{~mL} \mathrm{~L}^{-1}$.

\begin{tabular}{|c|c|c|c|c|c|c|}
\hline \multicolumn{2}{|l|}{ Treatments } & \multirow{2}{*}{$\begin{array}{l}\text { Vase life } \\
\text { (d) }\end{array}$} & \multirow{2}{*}{$\begin{array}{c}\text { Initial fresh } \\
\text { weight } \\
\text { (g) }\end{array}$} & \multirow{2}{*}{$\begin{array}{c}\text { Termination fresh } \\
\text { weight }(\mathrm{g})\end{array}$} & \multirow[t]{2}{*}{ Dry weight (g) } & \multirow{2}{*}{$\begin{array}{l}\text { Fresh weight } \\
\text { change } \\
\text { (g) }\end{array}$} \\
\hline Strain & $\begin{array}{c}\text { Conc. } \\
\left(\mathbf{m L ~ L ~} \mathbf{L}^{-1}\right)\end{array}$ & & & & & \\
\hline Tap water (-ve control) & - & $9.5^{z}$ & 77.1 & 73.2 & 8.2 & -3.9 \\
\hline \multirow[t]{3}{*}{ Nutrient Broth (+ve control) } & 5 & 9.5 & 76.8 & 73.5 & 8.3 & -3.4 \\
\hline & 10 & 8.8 & 79.4 & 77.6 & 8.4 & -1.8 \\
\hline & 20 & 9.3 & 82.5 & 79.6 & 8.7 & -2.9 \\
\hline \multirow[t]{3}{*}{ Pseudomonas fluorescens (PF-279) } & 5 & 8.9 & 84.4 & 78.5 & 8.3 & -5.9 \\
\hline & 10 & 9.4 & 80.2 & 77.3 & 8.5 & -2.9 \\
\hline & 20 & 9.1 & 82.4 & 76.4 & 8.2 & -6.0 \\
\hline \multirow[t]{3}{*}{ Pseudomonas fluorescens (PF-417) } & 5 & 9.1 & 81.4 & 78.1 & 8.7 & -3.3 \\
\hline & 10 & 9.4 & 79.4 & 71.0 & 8.0 & -6.2 \\
\hline & 20 & 9.0 & 77.2 & 75.2 & 8.4 & -4.2 \\
\hline \multirow[t]{3}{*}{ Bacillus pumilus } & 5 & 9.3 & 83.6 & 80.9 & 8.6 & -2.6 \\
\hline & 10 & 9.3 & 71.1 & 66.6 & 7.7 & -4.5 \\
\hline & 20 & 9.1 & 78.2 & 76.9 & 8.4 & -1.3 \\
\hline \multirow[t]{3}{*}{ Herbasperillum sp. } & 5 & 9.6 & 71.8 & 64.4 & 7.8 & -7.4 \\
\hline & 10 & 9.2 & 85.8 & 77.8 & 8.8 & -8.0 \\
\hline & 20 & 9.4 & 77.5 & 72.1 & 8.3 & -5.4 \\
\hline \multirow[t]{3}{*}{ Delftia acidovorans } & 5 & 9.4 & 75.0 & 74.6 & 7.9 & -0.3 \\
\hline & 10 & 9.1 & 74.6 & 73.6 & 7.9 & -1.0 \\
\hline & 20 & 9.0 & 80.1 & 75.6 & 8.3 & -4.5 \\
\hline \multicolumn{7}{|l|}{ Significance ${ }^{y}$} \\
\hline Overall & & NS & NS & NS & NS & 0.012 \\
\hline Strain (S) & & NS & NS & NS & NS & NS \\
\hline Conc. (C) & & NS & NS & NS & NS & NS \\
\hline $\mathrm{S} \times \mathrm{C}$ & & NS & NS & NS & NS & NS \\
\hline
\end{tabular}

Data represent means of five vases of three stems each or 15 individual stems; ${ }^{\mathrm{z}}$ Mean separation within columns by LSD at $P \leq 0.05$; ${ }^{\mathrm{y}} P$ values were obtained using General Linear Models (GLM) procedures (version 9.3; SAS Inst. Inc., Cary, $\mathrm{NC}$ ) for significant effects of different strains and their concentrations; ${ }^{\text {NS }}$ Nonsignificant at $P>0.05$. 
and nutrient broth at 5,10 , or $20 \mathrm{~mL} \mathrm{~L}^{-1}$ along with tap water (control). Each treatment was applied to five vases of three stems each.

Measurements: Data were recorded for vase life [time period (days) from placing stems in vases to the time of stem termination], initial and final fresh weight (one designated stem from each vase), dry weight (measured at termination after drying in oven at $70^{\circ} \mathrm{C}$ for $72 \mathrm{~h}$ ), fresh weight change, water uptake (measured in milliliters from all vases when first stem was terminated in entire experiment), percentage of florets opened during vase period, initial and final $\mathrm{pH}, \mathrm{pH}$ change, initial and final EC, EC change, and symptoms of termination, which included bent stem or petal wilting (Saleem et al., 2013). Cut stems were observed every day during vase period and every stem was terminated if it had developed one or more of the above mentioned symptoms on more than half of the flowers/petals, foliage, or stem (Ahmad et al., 2013a).

Statistical analysis: The experimental layout was completely randomized design with factorial arranged treatments and five replicate vases of three stems each. Data were analyzed using analysis of variance (ANOVA) and General Linear Models procedures of SAS (version 9.3, SAS Inst., Inc., Cary, NC) and Fisher's LSD at $\mathrm{P} \leq 0.05$ was used to separate means (Steel et al., 1997).

\section{RESULTS AND DISCUSSION}

Use of bio-control strains did not extend vase life of gladiolus stems and resulted in vase life similar to the stems placed in tap water (Table 1). Moreover, all stems had similar fresh weight changes during vase period and dry weight of a stem. However, stems placed in vase solutions with Pseudomonas florescens (PF-279) at $5 \mathrm{~mL} \mathrm{~L}^{-1}$ maintained water uptake and had a higher uptake than stems placed in solutions with Herbasperillum sp. or Pseudomonas florescens (PF-417) at 10 or $20 \mathrm{~mL} \mathrm{~L}^{-1}$ (Table 2). Use of higher concentrations of all bacterial strains tested lowered the water uptake, but had no effect on opening of the florets during vase period. In another study, bacterial strains viz. P. fulva and Escherichia coli K12 extended vase life compare to other tested bacterial strains, nutrient broth and control (DI), but all bacterial strains had no effect on water uptake of cut stems of zinnia (Carlson et al., 2015).

Table 2. Effect of different concentrations of various bacterial strains on water uptake, number of opened florets, and pH changes of 'Mammoth' gladiolus. Stems were placed in either tap water, pure nutrient broth, or in nutrient broth plus Pseudomonas fluorescens (PF-279), Pseudomonas fluorescens (PF-417), Bacillus pumilus, Herbasperillum sp., or Delftia acidovorans, at 5, 10, or $20 \mathrm{~mL} \mathrm{~L}{ }^{-1}$.

\begin{tabular}{|c|c|c|c|c|c|c|}
\hline \multicolumn{2}{|l|}{ Treatments } & \multirow{2}{*}{$\begin{array}{l}\text { Water uptake } \\
(\mathrm{mL})\end{array}$} & \multirow{2}{*}{$\begin{array}{l}\text { Number of opened } \\
\text { florets }(\%)\end{array}$} & \multirow[t]{2}{*}{ Initial pH } & \multirow[t]{2}{*}{ Final pH } & \multirow[t]{2}{*}{ pH change } \\
\hline Strains & $\begin{array}{l}\text { Conc. } \\
\left(\mathrm{mL} \mathrm{L} \mathbf{L}^{-1}\right)\end{array}$ & & & & & \\
\hline Tap water (-ve control) & - & $309.0 \mathrm{ab}$ & $70.8 \mathrm{abc}$ & $5.3 \mathrm{~h}$ & $4.9 \mathrm{e}$ & $-0.31 \mathrm{a}$ \\
\hline \multirow[t]{3}{*}{ Nutrient Broth (+ve control) } & 5 & $299.0 \mathrm{abc}$ & $76.8 \mathrm{a}$ & $8.0 \mathrm{~g}$ & $6.2 \mathrm{abc}$ & $-1.76 b c$ \\
\hline & 10 & $292.0 \mathrm{abc}$ & 69.3 bcde & $8.1 \mathrm{f}$ & $6.1 \mathrm{abc}$ & $-1.99 \mathrm{~cd}$ \\
\hline & 20 & $272.0 \mathrm{bcd}$ & $64.1 \mathrm{e}^{-10}$ & $8.1 \mathrm{f}$ & $6.2 \mathrm{abc}$ & $-1.91 \mathrm{bcd}$ \\
\hline \multirow[t]{3}{*}{ Pseudomonas fluorescens (PF-279) } & 5 & $327.0 \mathrm{a}$ & $70.6 \mathrm{abc}$ & $8.4 \mathrm{c}$ & $5.8 \mathrm{~d}$ & $-2.6 \mathrm{~h}$ \\
\hline & 10 & $293.0 \mathrm{abc}$ & $64.3 \mathrm{de}$ & $8.4 \mathrm{c}$ & $6.1 \mathrm{abc}$ & -2.30 efg \\
\hline & 20 & 282.0 abcd & $73.3 \mathrm{ab}$ & $8.1 \mathrm{~g}$ & $6.3 \mathrm{a}$ & $-1.71 b$ \\
\hline \multirow{3}{*}{ Pseudomonas fluorescens (PF-417) } & 5 & 286.0 abcd & $72.0 \mathrm{abc}$ & $8.6 \mathrm{a}$ & $6.1 \mathrm{abc}$ & $-2.53 \mathrm{gh}$ \\
\hline & 10 & $257.0 \mathrm{cde}$ & 68.3 bcde & $8.4 \mathrm{c}$ & $6.1 \mathrm{abc}$ & $-2.33 \mathrm{efg}$ \\
\hline & 20 & $243.0 \mathrm{de}$ & $66.8 \mathrm{cde}$ & $8.4 \mathrm{c}$ & $6.2 \mathrm{abc}$ & -2.24 ef \\
\hline \multirow[t]{3}{*}{ Bacillus pumilus } & 5 & $277.0 \mathrm{bcd}$ & 69.3 bcde & $8.0 \mathrm{~g}$ & $6.1 \mathrm{abc}$ & $-1.92 \mathrm{bcd}$ \\
\hline & 10 & $277.0 \mathrm{bcd}$ & $70.4 \mathrm{bcd}$ & $8.3 \mathrm{~d}$ & $6.1 \mathrm{abc}$ & $-2.23 \mathrm{de}$ \\
\hline & 20 & $289.0 \mathrm{abcd}$ & $71.5 \mathrm{abc}$ & $8.2 \mathrm{e}$ & $6.2 \mathrm{abc}$ & $-1.96 \mathrm{~cd}$ \\
\hline \multirow[t]{3}{*}{ Herbasperillum sp. } & 5 & $293.0 \mathrm{abc}$ & 70.2 bcde & $8.5 \mathrm{~b}$ & $6.0 \mathrm{~cd}$ & $-2.48 \mathrm{fgh}$ \\
\hline & 10 & $217.0 \mathrm{e}$ & $71.5 \mathrm{abc}$ & $8.5 \mathrm{~b}$ & $6.1 \mathrm{abc}$ & $-2.37 \mathrm{efgh}$ \\
\hline & 20 & 261.0 cde & 68.0 bcde & $8.4 \mathrm{c}$ & $6.3 \mathrm{ab}$ & $-2.14 \mathrm{de}$ \\
\hline \multirow[t]{3}{*}{ Delftia acidovorans } & 5 & $282.0 \mathrm{abcd}$ & 70.0 bcde & $8.5 \mathrm{~b}$ & $6.1 \mathrm{abc}$ & -2.37 efgh \\
\hline & 10 & $269.0 \mathrm{bcd}$ & $73.0 \mathrm{abc}$ & $8.4 \mathrm{c}$ & $6.0 \mathrm{bc}$ & -2.35 efg \\
\hline & 20 & $281.0 \mathrm{abcd}$ & 70.2 bcde & $8.2 \mathrm{e}$ & $6.2 \mathrm{abc}$ & $-1.99 \mathrm{~cd}$ \\
\hline \multicolumn{7}{|l|}{ Significance ${ }^{y}$} \\
\hline Overall & & NS & NS & $<0.0001$ & $<0.0001$ & $<0.0001$ \\
\hline Strain $(\mathrm{S})$ & & 0.0189 & NS & $<0.0001$ & NS & $<0.0001$ \\
\hline Conc. (C) & & 0.0138 & NS & $<0.0001$ & $<0.0001$ & $<0.0001$ \\
\hline $\mathrm{S} \times \mathrm{C}$ & & NS & 0.004 & $<0.0001$ & 0.006 & $<0.0001$ \\
\hline
\end{tabular}

Data represent means of five vases of three stems each or 15 individual stems; ${ }^{\mathrm{z}}$ Mean separation within columns by LSD at $P \leq 0.05$; ${ }^{y} P$ values were obtained using General Linear Models (GLM) procedures (version 9.3; SAS Inst. Inc., Cary, NC) for significant effects of different strains and their concentrations; ${ }^{\text {NS }}$ Nonsignificant at $P>0.05$. 
Table 3. Bacterial populations of various bacterial strains, Pseudomonas fluorescens (PF-279), Pseudomonas fluorescens (PF-417), Bacillus pumilus, Herbasperillum sp., or Delftia acidovorans, sampled on day 0, 3, 6 or 9. Inoculum of each strain was added at 5,10 , or $20 \mathrm{~mL} \mathrm{~L}^{-1}$.

\begin{tabular}{|c|c|c|c|c|c|}
\hline \multirow[t]{2}{*}{ Strain } & \multirow{2}{*}{$\begin{array}{c}\text { Volume of inoculant } \\
\text { added }\left(\mathrm{mL} \mathrm{L}^{-1}\right)\end{array}$} & \multicolumn{4}{|c|}{ Bacterial counts (cfu $0.1 \mathrm{~mL}^{-1}$ ) } \\
\hline & & Day 0 & Day 3 & Day 6 & Day 9 \\
\hline \multirow{3}{*}{ Water (-ve control) } & & & $11 \times 10^{3}$ & $28 \times 10^{3}$ & $51 \times 10^{3}$ \\
\hline & & & $1 \times 10^{6}$ & $1 \times 10^{6}$ & - \\
\hline & & & - & - & - \\
\hline \multirow[t]{8}{*}{ Nutrient broth (+ve control) } & 5 & & $288 \times 10^{4}$ & $235 \times 10^{4}$ & $235 \times 10^{4}$ \\
\hline & & & - & $7 \times 10^{6}$ & $4 \times 10^{6}$ \\
\hline & & & - & - & - \\
\hline & 10 & & $366 \times 10^{4}$ & $112 \times 10^{4}$ & $187 \times 10^{4}$ \\
\hline & & & $6 \times 10^{6}$ & $10 \times 10^{6}$ & $2 \times 10^{6}$ \\
\hline & & & $\begin{array}{c}- \\
208 \times 10^{4}\end{array}$ & $\stackrel{-}{-}$ & - \\
\hline & 20 & & $298 \times 10^{4}$ & $225 \times 10^{4}$ & $133 \times 10^{4}$ \\
\hline & & & $53 \times 10$ & $32 \times 10^{\circ}$ & $1 \times 10$ \\
\hline \multirow[t]{9}{*}{ Pseudomonas fluorescens (PF-279) } & 5 & $156 \times 10^{2}$ & $287 \times 10^{4}$ & $170 \times 10^{4}$ & $210 \times 10^{4}$ \\
\hline & & $8 \times 10^{3}$ & $5 \times 10^{6}$ & $4 \times 10^{6}$ & $3 \times 10^{6}$ \\
\hline & & $1 \times 10^{4}$ & $1 \times 10^{8}$ & - & - \\
\hline & 10 & & $463 \times 10^{4}$ & $184 \times 10^{4}$ & $256 \times 10^{4}$ \\
\hline & & & $2 \times 10^{6}$ & $6 \times 10^{6}$ & $8 \times 10^{6}$ \\
\hline & & & $1 \times 10^{8}$ & - & - \\
\hline & 20 & & $612 \times 10^{4}$ & $186 \times 10^{4}$ & $120 \times 10^{4}$ \\
\hline & & & $2 \times 10^{6}$ & $5 \times 10^{6}$ & $2 \times 10^{6}$ \\
\hline & & & - & - & - \\
\hline \multirow[t]{9}{*}{ Pseudomonas fluorescens (PF-417) } & 5 & $356 \times 10^{2}$ & $201 \times 10^{4}$ & $105 \times 10^{4}$ & $31 \times 10^{4}$ \\
\hline & & $200 \times 10^{3}$ & $92 \times 10^{6}$ & $2 \times 10^{6}$ & $8 \times 10^{6}$ \\
\hline & & $53 \times 10^{4}$ & - & - & - \\
\hline & 10 & & $290 \times 10^{4}$ & $131 \times 10^{4}$ & $107 \times 10^{4}$ \\
\hline & & & $5 \times 10^{6}$ & $1 \times 10^{6}$ & $4 \times 10^{6}$ \\
\hline & & & $1 \times 10^{8}$ & - & - \\
\hline & 20 & & $415 \times 10^{4}$ & $152 \times 10^{4}$ & $203 \times 10^{4}$ \\
\hline & & & $6 \times 10^{6}$ & $2 \times 10^{6}$ & $4 \times 10^{6}$ \\
\hline & & & $1 \times 10^{8}$ & - & - \\
\hline \multirow[t]{9}{*}{ Bacillus pumilus } & 5 & $413 \times 10^{2}$ & $136 \times 10^{4}$ & $180 \times 10^{4}$ & $216 \times 10^{4}$ \\
\hline & & $60 \times 10^{3}$ & $11 \times 10^{6}$ & $2 \times 10^{6}$ & $3 \times 10^{6}$ \\
\hline & & $8 \times 104$ & $1 \times 10^{8}$ & - & - \\
\hline & 10 & & $313 \times 10^{4}$ & $224 \times 10^{4}$ & $400 \times 10^{4}$ \\
\hline & & & $15 \times 10^{6}$ & $18 \times 10^{6}$ & $19 \times 10^{6}$ \\
\hline & & & - & - & $1 \times 10^{8}$ \\
\hline & 20 & & $246 \times 10^{4}$ & $120 \times 10^{4}$ & $109 \times 10^{4}$ \\
\hline & & & $10 \times 10^{6}$ & $7 \times 10^{6}$ & $11 \times 10^{6}$ \\
\hline & & & $1 \times 10^{8}$ & - & - \\
\hline \multirow[t]{9}{*}{ Herbasperillum $\mathrm{sp}$. } & 5 & $456 \times 10^{2}$ & $280 \times 10^{4}$ & $35 \times 10^{4}$ & $285 \times 10^{4}$ \\
\hline & & $90 \times 10^{3}$ & $42 \times 10^{6}$ & $1 \times 10^{6}$ & $7 \times 10^{6}$ \\
\hline & & $22 \times 10^{4}$ & $2 \times 10^{8}$ & - & - \\
\hline & 10 & & $495 \times 10^{4}$ & $186 \times 10^{4}$ & $149 \times 10^{4}$ \\
\hline & & & $43 \times 10^{6}$ & $4 \times 10^{6}$ & $73 \times 10^{6}$ \\
\hline & & & $4 \times 10^{8}$ & - & - \\
\hline & 20 & & $510 \times 10^{4}$ & $81 \times 10^{4}$ & $35 \times 10^{4}$ \\
\hline & & & $50 \times 10^{6}$ & $1 \times 10^{6}$ & $1 \times 10^{6}$ \\
\hline & & & $8 \times 10^{8}$ & - & - \\
\hline \multirow{9}{*}{ Delftia acidovorans } & 5 & $296 \times 10^{2}$ & $184 \times 10^{4}$ & $290 \times 10^{4}$ & $288 \times 10^{4}$ \\
\hline & & $47 \times 10^{3}$ & $2 \times 10^{6}$ & $8 \times 10^{6}$ & $18 \times 10^{6}$ \\
\hline & & $11 \times 10^{4}$ & - & - & \\
\hline & 10 & & $416 \times 10^{4}$ & $556 \times 10^{4}$ & $607 \times 10^{4}$ \\
\hline & & & $13 \times 10^{6}$ & $43 \times 10^{6}$ & $29 \times 10^{6}$ \\
\hline & & & & & \\
\hline & 20 & & $640 \times 10^{4}$ & $240 \times 10^{4}$ & $210 \times 10^{4}$ \\
\hline & & & $23 \times 10^{6}$ & $5 \times 10^{6}$ & $11 \times 10^{6}$ \\
\hline & & & $2 \times 10^{8}$ & - & - \\
\hline
\end{tabular}

Values represent means of samples from two or three vases.

During vase period, $\mathrm{pH}$ of the solutions containing bacterial strains became much more acidic with the greatest decrease (2.6 units) for PF-279 at $5 \mathrm{~mL} \mathrm{~L}^{-1}$, which might be the reason for continued water uptake by the stems (Table 2). Higher water uptake due to low $\mathrm{pH}$ has also been reported in other studies with different ornamental species (Ahmad et al., 2013b; Carlson and Dole, 2013). However, the solution EC 
changes for all treatments were statistically similar (data not presented).

The bacterial population in different vase solutions greatly varied when sampled on 3 day intervals during vase life (Table 3). Although no significant extension in vase life of cut gladiolus stems occurred, the PF-279 bacteria appeared to have countered the negative effects of detrimental bacteria in the vase solutions to produce similar vase life as with tap water. It has been reported that avoiding bacterial proliferation in vase solutions is more important as compared to their populations in the solutions (Carlson et al., 2015). Certain bacterial strains may have little or no effect but several species may shorten the vase life of various cut flower crops (van Doorn et al., 1991; Jacob and Kim, 2010) by blocking the vascular system (Put, 1990), by producing enzymes which kill plant tissues (Membre and Burlot, 1994), or by producing senescence causing hormones, such as ethylene (van Doorn et al., 1991). Therefore, more comprehensive studies need to be conducted by using different strains, concentrations and populations of the bacteria for evaluation of their effects on extending postharvest longevity of cut flowers.

In summary, Pseudomonas florescens (PF-279) yielded better results among all tested strains by maintaining water uptake by the stems (Gast, 2000) due to low solution $\mathrm{pH}$, which is beneficial in extending vase life of many cut flower species by slowing bacterial growth in vase solution (van Doorn and Perik, 1990), avoiding stem vasculature blockage and embolisms (Durkin, 1979), maintaining higher stem hydraulic conductance (Marousky, 1971), reducing water stress symptoms and numerically lengthening the vase life of cut gladiolus stems.

Acknowledgements: The authors would like to acknowledge the Glad-A-Way Farms, CA, USA, for providing cut gladiolus stems, and Washington State University, WA, USA, for providing bacterial strain, Pseudomonas fluorescens (PF279), for the study.

\section{REFERENCES}

Ahmad, I., J.M. Dole, M. Saleem, M.A. Khan, A. Akram and A.S. Khan. 2013a. Preservatives and packaging material have an impact on the post-harvest longevity of cut Rosa hybrida L. 'Kardinal' flowers. J. Hort. Sci. Biotech. 88:251-256.

Ahmad, I., J.M. Dole, A.S. Carlson and F.A. Blazich. 2013b. Water quality affects vase life of cut callas, hydrangeas and snapdragons. Sci. Hortic. 153:26-33.

Ahmad, I., J.M. Dole, E.M.R. Clark and F.A. Blazich. 2014. Floral foam and/or conventional or organic preservatives affect the vase-life and quality of cut rose (Rosa $\times$ hybrida L.) stems. J. Hort. Sci. Biotech. 89:41-46.
Carlson, A.S. and J.M. Dole. 2013. Postharvest water quality affects vase life of cut Dendranthema, Dianthus, Helianthus, and Zinnia. Sci. Hortic. 164:277-286.

Carlson, A.S., J.M. Dole, A.G. Matthysse, W.A. Hoffmann and J.L. Kornegay. 2015. Bacteria species and solution $\mathrm{pH}$ effect postharvest quality of cut Zinnia elegans. Sci. Hortic. 194:71-78.

Duffy, B.K., A. Simon and D.M. Weller. 1996. Combination of Trichoderma koningii with Fluorescent pseudomonads for control of take-all on wheat. Phytopath. 86:188-194.

Durkin, D. 1979. Effect of millipore filtration, citric acid, and sucrose on peduncle water potential of cut rose flower. J. Amer. Soc. Hort. Sci. 104:860-863.

Gast，K.L.B. 2000. Water quality for florists-why is it so important. Kans. State Univ. Agr. Expt. Sta. Coop. Ext. Serv., USA.

Jacob, B.M. and E. Kim. 2010. Inhibiting biofilm formation of Enterobacter sp. prevented premature withering in cut flowers. Korean J. Chem. Eng. 27:1252-1257.

Janisiewicz, W.J. 1996. Ecological diversity, niche overlap, and coexistence of antagonists used in developing mixtures for biocontrol of post-harvest diseases of apples. Phytopath. 86:473-479.

Janisiewicz, W.J. and B. Bors. 1995. Development of microbial community of bacterial and yeast antagonists to control wound-invading postharvest pathogens of fruits. Appl. Environ. Microbiol. 61:3261-3267.

Jetiyanon, K. and J.W. Kloepper. 2002. Mixtures of plant growth promoting rhizobacteria for induction of systemic resistance against multiple plant diseases. BioControl 24:285-291.

Kloepper, J.W., C.M. Ryu and S. Zhang. 2004. Induced systemic resistance and promotion of plant growth by Bacillus spp. Phytopath. 94:1259-1266.

Marousky, F.J. 1971. Inhibition of vascular blockage and increased moisture retention in cut roses induced by $\mathrm{pH}$, 8-hydroxyquinoline citrate and sucrose. J. Am. Soc. Hort. Sci. 96:38-41.

Membre, J.M. and P.M. Burlot. 1994. Effects of temperature, $\mathrm{pH}$, and $\mathrm{NaCl}$ on growth and pectinolytic activity of Pseudomonas marginalis. Appl. Environ. Microbiol. 60:2017-2022.

Paulitz, T.C., J.S. Ahmad and R. Baker. 1990. Integration of Pythium nunn and Trichoderma harzianum isolate T-95 for the biological control of Pythium damping-off of cucumber. Plant Soil 121:243-250.

Put, H.M.C. 1990. Micro-organisms from freshly harvested cut flower stems and developing during the vase life of chrysanthemum, gerbera and rose cultivars. Sci. Hortic. 43:129-144.

Raupach, G.S. and J.W. Kloepper. 1998. Mixtures of plant growth promoting rhizobacteria enhance biological 
control of multiple cucumber pathogens. Phytopath. 88:1158-1164.

Sajjad, Y., M.J. Jaskani, M.Y. Ashraf, M. Qasim and R. Ahmad. 2014. Response of morphological and physiological growth attributes to foliar application of plant growth regulators in gladiolus 'white prosperity'. Pak. J. Agri. Sci. 51:123-129.

Saleem, M., I. Ahmad and M.A. Khan. 2013. Cultivar effects on growth, yield and cormel production of gladiolus (Gladiolus grandiflorus L.). J. Ornam. Hort. Pl. 3:39-48.

Shanmugam, V., N. Kanoujia, M. Singh, S. Singh and R. Prasad. 2011. Biocontrol of vascular wilt and corm rot of gladiolus caused by Fusarium oxysporum f. sp. gladioli using plant growth promoting rhizobacterial mixture. Crop Prot. 30:807-813.

Shanmugam, V., N. Senthil, T. Raguchander, A. Ramanathan and R. Samiyappan. 2002. Interaction of Pseudomonas fluorescens with Rhizobium for their effect on the management of peanut root rot. Phytoparasitica 30:169176.

Steel, R.G.D., J.H. Torrie and D.A. Dickey. 1997. Principles and Procedures of Statistics: A biometrical approach. McGraw Hill Book Co., New gladiolYork, USA.

Teixeira-da-Silva, J.A. 2003. The cut flower: postharvest considerations. Online J. Biol. Sci. 3:406-442.

van Doorn, W.G. and R.R.J. Perik. 1990. Hydroxyquinoline citrate and low $\mathrm{pH}$ prevent vascular blockage in stems of cut rose flowers by reducing the number of bacteria. J. Amer. Soc. Hort. Sci. 115:979-981.

van Doorn, W.G., H.C.M. de Stigter, Y. de Witte and A. Boekestein. 1991. Micro-organisms at the cut surface and in xylem vessels of rose stems: a scanning electron microscopy study. J. Appl. Bacteriol. 70:34-39.

van Loon, L.C., P.A.H.M. Bakker and C.M.J. Pieterse. 1998. Systemic resistance induced by rhizosphere bacteria. Ann. Rev. Phytopathol. 36:453-483. 